\title{
PERFORMANCE ANALYSIS OF ELECTRONIC MECHANICAL ASSISTED BRAKING OF ENVIRONMENTAL-FRIENDLY VEHICLES BASED ON PEDAL DECOUPLING
}

\author{
Yungui Wei \\ Shanxi Traffic Vocational and Technical College, Taiyuan 030031, China. \\ Email: weiyunguity@yeah.net
}

\begin{abstract}
This research aims to reduce the impact of the regenerative braking force generated by the new energy vehicle on the braking performance during regenerative braking. It mainly focuses on the braking design scheme of pedal decoupling and the corresponding pedal decoupling strategy. First, the pedal decoupling scheme is designed, and the electronic mechanical assisted braking model and the overall model of the driving vehicle are established. The model includes electromechanical booster model, active accumulator model, and hydraulic braking model. The electromechanical booster model mainly includes the permanent magnet synchronous motor model and the transmission mechanism model. Then, the pedal decoupling strategy based on the new decoupling scheme is studied, and the decoupling process is divided into three stages. According to the control requirements of each stage, the control of pressure reducing valve and the pump motor in the electronic stability program (ESP) is realized, so as to adjust the brake circuit hydraulic pressure during the decoupling process. Moreover, the decoupling current is calculated to adjust the torque of the electromechanical booster motor, so as to adjust the pedal feel. Finally, the control strategy of the electro-mechanical assist motor is designed to realize the control of the motor. The experimental results show that the designed decoupling strategy can make the master cylinder hydraulic pressure change according to the distribution result of the braking force distribution strategy under the three braking strengths of low, medium, and high, which has a good decoupling effect. In addition, the decoupling electro-hydraulic compound braking realizes the precise control of the wheel cylinder pressure and effectively recovers the braking energy. The braking energy feedback efficiency reaches $54.8 \%$. The designed decoupling strategy provides some references for the adoption of pedal decoupling in automobile assisted braking.
\end{abstract}

Keywords: Electronic Mechanical Assisted Braking; Pedal Decoupling; New Energy Vehicles; Active Accumulators.

\section{Introduction}

Since the 21st century, China's new energy vehicle industry has been greatly developed, and the sales and ownership of new energy vehicles continue to increase. Compared with traditional vehicles, new energy vehicles not only have a greater advantage in energy emission reduction, but also can further improve the effect of energy conservation and emission reduction by using constantly updated technologies [1]. Regenerative braking is a key technology in the field of energy saving and emission reduction. Regenerative braking for new energy vehicles can effectively recover braking energy, improves energy utilization, further improves the range of new energy vehicles, and then improves the economy of the vehicle. The working principle of regenerative braking is making use of the feedback braking force of the motor.
When the vehicle needs to brake, the driving shaft is pulled back to drive the motor to run and make it run into the generator state and then output electric power, charging the energy storage battery, and finally recover the braking energy. To ensure the safety of the vehicle and the driver, it is necessary to modify the hydraulic braking force and the distribution relationship between the front and rear axles of the vehicle after the introduction of regenerative braking force $[2,3]$. The usual approach is designing an electro-hydraulic braking force distribution strategy to realize the redistribution of the vehicle's front and rear axle braking and the electro-hydraulic braking force of the drive shaft. Then, the coordination of regenerative braking and hydraulic braking is realized. However, from the law of conservation of energy, when the total braking force remains unchanged, the redistributed braking force will cause the original hydraulic braking force 
distribution relationship to change. In the current vehicle braking, the brake pedal is coupled with the wheel cylinder, so the change of hydraulic braking force will cause the change of the pedal feeling of the driver. The coupling relationship between brake pedal and the wheel cylinder is cut off during the regenerative braking process to deal with the feel change of driver's pedal. Generally, enterprises will design new braking deployment schemes to enable the hydraulic circuit to realize the hydraulic adjustment function and maintain the pedal feel unchanged during the decoupling process.

In this research context, many researchers constructed different degrees of braking deployment schemes to achieve decoupling. Kwon et al. (2019) [4] pointed out that electrical and mechanical braking was a motor-based braking force generation module. Various sensors such as motor position, motor current, and clamping force sensor are needed to achieve stable vehicle deceleration control. However, the failure of the sensor may lead to a decrease in performance, so monitoring is essential. For this reason, a new electrical and mechanical brake (EMB) model is proposed, which divides the clamping force term into two parts: linear compensation and nonlinear compensation, and it employs Kalman filtering algorithm to design the state estimator. The model is simple lineament, and the Kalman filtering algorithm has strong robustness to noise, which helps to improve the accuracy of state estimation.

Finally, the effectiveness of the method was verified by the experiment on the EMB test bench. Sheng et al. (2018) [5] introduced the basic concept, structure, and importance of the coordinated control of EMB of vehicles, and analysed the current research status and future trends of electromechanical braking. In the proposed hydraulic-pneumatic hybrid power model, Wang et al. (2018) Bravo et al. (2018) [6] proposed that braking energy should be recovered by the hydraulic system and stored in hydraulic accumulators and air accumulators. When the hydraulic system and the internal combustion engine shared vehicle propulsion in parallel, compressed air was used to assist auxiliary machinery. The author verified his idea with the lumped parameter simulation model written by MATLAB/Simulink, and verified it by comparing the measurement results and prediction results from the experiment. Peng et al. (2018) [7] established an EMB model and a semi-vehicle dynamics model. Aiming at the nonlinearity and uncertainty of the EMB model, a fuzzy sliding mode control strategy based on the wheel-to-slip ratio of the EMB model is proposed, which also considers the normal and emergency braking states of the EMB model.

According to the changes of the front and rear axle loads during braking, the equivalent control law of the sliding mode controller is designed, and the switching control law is adjusted through the fuzzy corrector. The results show that compared with proportional-integral-derivative (PID) control and sliding mode control, the fuzzy sliding mode control strategy has better performance and adaptability to various roads. It can make the target value faster and smoother, and has a strong anti-interference ability. Fuzzy sliding mode control has a positive effect on improving the braking stability of vehicles. However, the China market has not been commercialized in terms of new type of boosting products, and the market is mainly monopolized by foreign companies. The research and design of electronic mechanical assisted braking is of good market prospect and adoption value.

In this research, the performance of electronic mechanical assisted braking of new energy vehicles and the design of pedal decoupling scheme are studied. An electronic mechanical assisted braking model and a vehicle model are established. Then, the pedal decoupling strategy based on the new decoupling scheme is studied, and the decoupling strategy is verified by simulation.

\section{Decoupling Electro - Hydraulic Compound Braking and Braking Force Distribution Control Strategy 2.1 Decoupling Electro-Hydraulic Composite Braking}

Since the decoupling compound braking has the advantages of high feedback effect, good pedal feel, and convenient for coordinated control with the regeneration system, an integrated active brake decoupling compound braking is designed based on decoupling compound braking. The model consists of ten modules, which are brake pedal, integrated brake master cylinder, hydraulic adjustment unit, battery pack and battery management, electric motor and electric motor controller, transmission system, and composite brake unit $[8,9]$. The abovementioned modules are classified into two large sections, namely the hydraulic brake subsystem and the regenerative brake subsystem. The hydraulic brake subsystem uses an integrated brake master cylinder to decouple the pressure of the brake pedal and the cylinder $[10,11]$. The vehicle controller and hydraulic control unit collect the information of the two systems in real time and send the information to the compound brake control unit. The task of the compound brake control unit is collecting brake pedal information, and analysing the driver's braking demand from the collected brake pedal information. According to the different operating conditions of different vehicles, the front and rear axles and electric and hydraulic braking forces of the vehicles are allocated, and the wheel cylinder hydraulic 
pressure and regenerative braking commands are sent to the hydraulic adjustment unit and the vehicle controller, respectively.
The decoupling electro-hydraulic compound braking structure is shown in Figure 1, and the hydraulic brake model is shown in Figure 2.

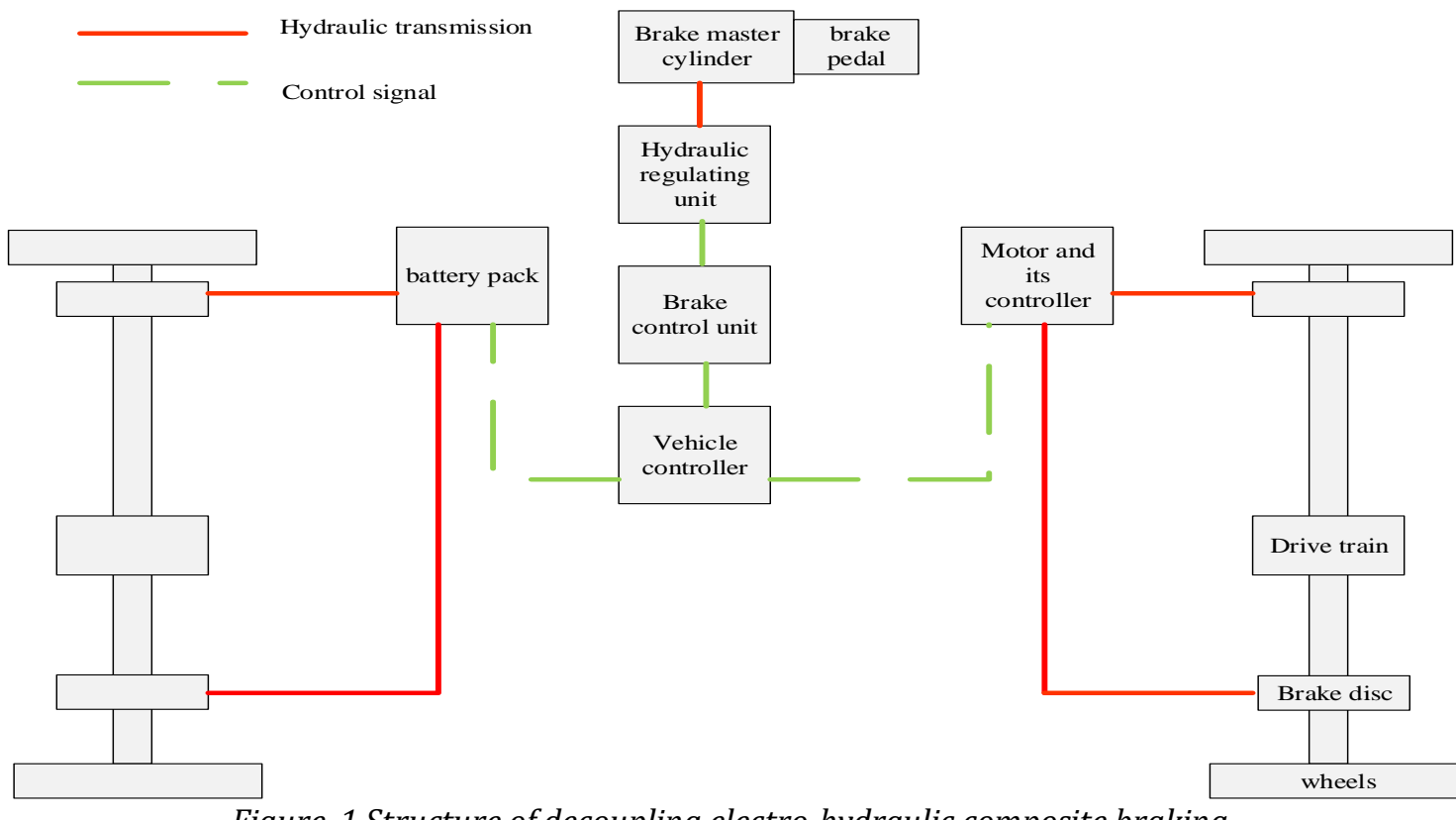

Figure. 1 Structure of decoupling electro-hydraulic composite braking.

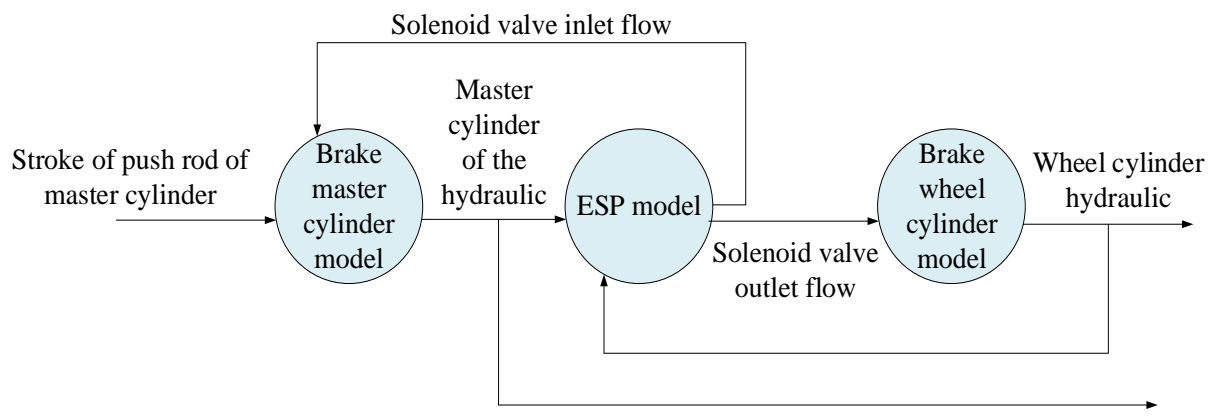

Figure. 2 Block diagrams of hydraulic braking model.

\subsection{Composition of the Integrated Brake Master Cylinder}

The brake master cylinder used in the research is a plunger-type master cylinder, which is composed of two chambers. The chamber closer to the pedal push rod is called the first chamber, and vice versa is called the second chamber. The corresponding piston in the first chamber is also called the first piston $[12,13]$. Generally speaking, the first chamber and the second chamber can work separately to produce the same brake hydraulic pressure in their respective circuits. When one chamber fails, the brake circuit of the other chamber can still work normally and generate hydraulic pressure. The actions of the master cylinder are divided into three stages. In the first stage, the first piston moves together with the first piston under the action of the push rod of the master cylinder. Pressure is generated in the second chamber. As the hydraulic pressure continues to increase, the first and second pistons move separately and enter the second stage. Then, the two pistons generate brake hydraulic pressures with very small differences in their respective chambers. When the return springs of the two pistons are compressed to the limit, it enters the third stage. At this time, the first chamber continues to pressurize, and the hydraulic pressure of the second chamber only changes slightly $[14,15]$. The integrated brake master cylinder is the most core design link in the entire decoupling electro-hydraulic composite braking. It forms an integrated brake assembly with high-pressure accumulators, hydraulic pumps, etc., After the system is powered on, the composite control unit controls the hydraulic pump to maintain the pressure of the high-pressure accumulator according to the feedback signal of the hydraulic sensor. When the brake pedal is depressed, the second piston in the second chamber of the hydraulic booster remains stationary, Then, the pedal is driven by the reaction force of the spring to brake. After the use of the brake pedal, the 
accumulator controls the brake pressure of different wheel cylinders individually according to the action control command. When there is an electrical failure, the accumulator no longer maintains high voltage $[16,17]$.

\subsection{Braking Force Distribution and Control Strategy}

In the process of decoupling electro-hydraulic composite braking design, to make the vehicle stable, it is necessary to implement a braking force distribution control strategy that is restricted by regulations, electric motors, and battery pack characteristics [18]. In addition to the abovementioned constraints, the electric motor and battery pack also have a certain constraint on the system. Clarifying the internal environment of the vehicle system, analysing the factors that affect the feedback efficiency comprehensively, and allocating the vehicle braking function reasonably help implement the optimal control strategy of effective feedback power, which also enhance the feedback function of the vehicle to optimize the recovery of braking energy $[19,20]$.

\subsection{Restraint of Braking Regulations}

According to the requirements of relevant laws and regulations, the distribution of braking force between the axles of passenger vehicles meets the following requirements. In the case of all vehicle loads, when the braking strength $\alpha$ is between 0.15 and 0.8 , the rear axle adhesion coefficient should not be located above the front axle. When the adhesion coefficient $\Phi$ is between 0.2 and 0.8 , the braking strength satisfies the relationship shown in equation (1) [21]. When the braking intensity $\alpha$ is between 0.15 and 0.8 , the rear axle curve is below the curve shown in equation (2).

$$
\begin{aligned}
& \alpha \geq 0.1+0.7(\Phi-0.2) \\
& \alpha=0.9 \Phi
\end{aligned}
$$

\subsection{Motor Battery Constraints}

Drive motor and battery are important components of regenerative braking, and their characteristics also affect the distribution of regenerative braking force. When the speed of the passenger car is low, and the motor speed doesn't reach the minimum speed of the power generation state, regenerative braking is not performed [22,23]. When the motor speed is less than the base speed, the motor works in the constant torque zone, and when it is higher than the base speed, the motor works in the constant power zone. The external characteristics of the motor torque are shown in equation (3).

$$
T_{\mathrm{m}}=\left\{\begin{array}{cc}
0 & \left(\mathrm{n}_{m}<\mathrm{n}_{0}\right) \\
T_{\mathrm{m}-\max } & \left(\mathrm{n}_{0} \leq \mathrm{n}_{m} \leq \mathrm{n}_{1}\right) \\
\frac{9550 P_{\mathrm{m}-\max }}{\mathrm{n}_{\mathrm{m}}} & \left(\mathrm{n}_{m} \geq \mathrm{n}_{1}\right)
\end{array}\right.
$$

In equation (3), $T_{\mathrm{m}}$ is the torque that can be generated under the motor constraint characteristics, $T_{\mathrm{m} \text {-max }}$ is the maximum torque of the motor, $P_{\mathrm{m}-\max }$ is the peak power of the motor, $\mathrm{n}_{\mathrm{m}}$ is the motor speed, $\mathrm{n}_{0}$ is the start regenerative braking speed, and $\mathrm{n}_{1}$ is the base speed of the motor.

The power torque of the electric mechanism is also affected by the maximum charging power of the battery. When the maximum charging power of the battery is less than the generated power of the motor, the maximum charging power of the battery can determine the maximum torque of the motor [24], as shown in equation (4).

$$
T_{\mathrm{b}}=\left\{\begin{array}{cc}
0 & \left(\mathrm{n}_{m}<\mathrm{n}_{0}\right) \\
\frac{9550 P_{\mathrm{b}-\max }}{\mathrm{n}_{1}} & \left(\mathrm{n}_{0} \leq \mathrm{n}_{m} \leq \mathrm{n}_{1}\right) \\
\frac{9550 P_{\mathrm{m}-\max }}{\mathrm{n}_{\mathrm{m}}} & \left(\mathrm{n}_{m} \geq \mathrm{n}_{1}\right)
\end{array}\right.
$$

In equation (4), $T_{\mathrm{b}}$ is the torque generated under the battery constraint, and $P_{\mathrm{b}-\max }$ is the maximum charging power of the battery.

\subsection{Security and Compliance Constraints}

Vehicle speed, braking intensity, and state of charge (SOC) of battery all have an impact on regenerative braking. When the vehicle speed is low, the electromotive force generated by the motor is small, it is difficult to charge the battery, and it will reduce the reliability of braking when participating in regenerative braking. Therefore, regenerative braking doesn't work at low vehicle speeds [25,26]. When the braking intensity is too strong, regenerative braking also needs to stop working. When the SOC is too large, the battery capacity is close to saturation. To avoid overcharging, the braking capacity will no longer be recovered. Therefore, before the vehicle speed, braking intensity, and battery SOC exceed the threshold, the 
control regenerative braking force needs to be slowly reduced until it exits [27]. Therefore, to optimize braking and reduce impact, the vehicle speed influence factor is introduced as shown in equation (5), the braking intensity influence factor is shown in equation (6), and the battery influence factor is shown in equation (7)

$$
K_{v}=\left\{\begin{array}{cc}
0 & u_{a}<u_{\text {under }} \\
\frac{u_{\text {under }}-u_{a}}{u_{\text {under }}-u_{\text {upp }}} & u_{\text {under }} \leq u_{a} \leq u_{\text {upp }} \\
1 & u_{a}>u_{\text {upp }}
\end{array}\right.
$$

In equation (5), $\mathrm{u}_{\mathrm{a}}$ is the threshold, $U_{\text {under }}$ is the maximum value, and $U_{\text {under }}$ is the minimum value.

$$
K_{z}=\left\{\begin{array}{cc}
0 & z>z_{\text {upp }} \\
\frac{z_{\text {under }}-z}{z_{\text {under }}-z_{\text {upp }}} & \mathrm{z}_{\text {under }} \leq z \leq z_{\text {upp }} \\
1 & z<z_{\text {under }}
\end{array}\right.
$$

In equation (6), $z$ is the threshold, $z_{\text {under }}$ is the maximum value, and $z_{\text {upp }}$ is the minimum value.

$$
K_{\mathrm{soc}}=\left\{\begin{array}{cl}
0 & s O c>s O c_{u p p} \\
\frac{s O c_{u p p}-s O c}{s O c_{u p p}-S O c_{u n d e r}} & s O c_{u n d e r} \leq s O c \leq s O c_{u p p} \\
1 & s O c<s O c_{\text {under }}
\end{array}\right.
$$

In equation (7), $S O C$ is the threshold, $S O c_{\text {under }}$ is the maximum value, and $s o c_{u p p}$ is the minimum value.

The introduction of the factor makes the target regenerative braking force not abrupt with changes in vehicle speed, braking intensity, and battery SOC, ensuring braking safety and smoothness when regenerative braking exits the braking process. Figure 3 shows the change trend of the regenerative braking force restraint factor.

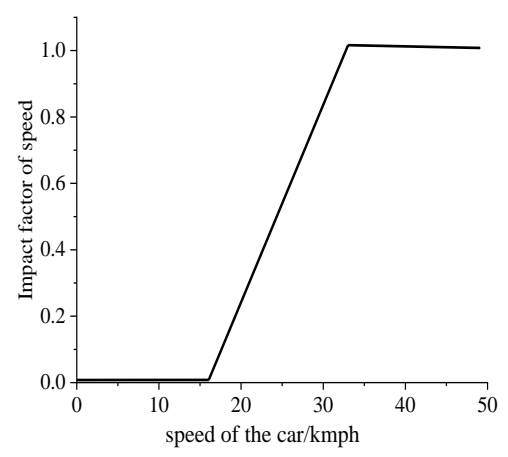

(a)

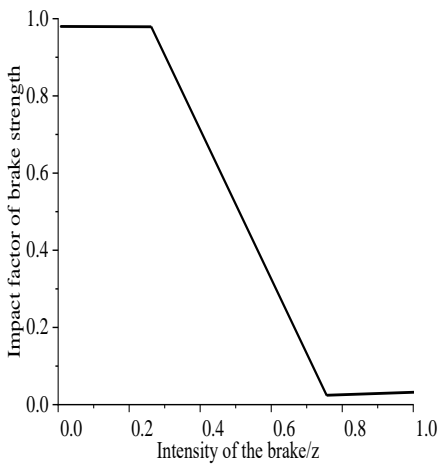

(b)

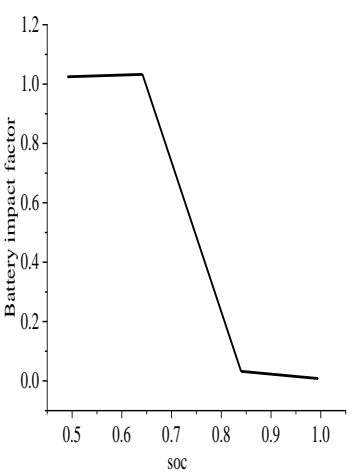

(c)

Figure. 3 Regenerative braking force constraints.

(a: vehicle speed influencing factor, b: braking intensity influencing factor, c: soc influencing factor.)

\subsection{Decoupling Strategy based on Electronic Analytical Booster}

Figure 4 shows the overall design block diagram based on the electronic mechanical booster decoupling strategy.

The specific process is divided into 4 steps, which are as follows.

1. An electro-hydraulic braking force distribution and control strategy is designed to distribute the vehicle's front and rear axle braking force and electro-hydraulic braking force. In this process, it is necessary to calculate the target value of regenerative braking force, the target value of hydraulic braking force, the threshold value of regenerative braking force, and the target hydraulic pressure of the wheel cylinder based on the pedal push rod stroke signal and vehicle state signal input.

2 . The pedal push rod stroke signal and the motor status signal that need to be accepted by the booster motor control strategy are taken as inputs, to obtain the control signal of the booster motor of the electronic mechanical booster.

3. The pedal decoupling strategy is adopted to obtain the control signal of the solenoid valve and the motor, and the obtained signal is utilized to adjust the hydraulic pressure of the brake circuit during decoupling, and then the pedal decoupling current is calculated and input to the control strategy of the booster motor to adjust the boost size.

4. The target value of regenerative braking force is regarded as the actual value and input into the vehicle model. 


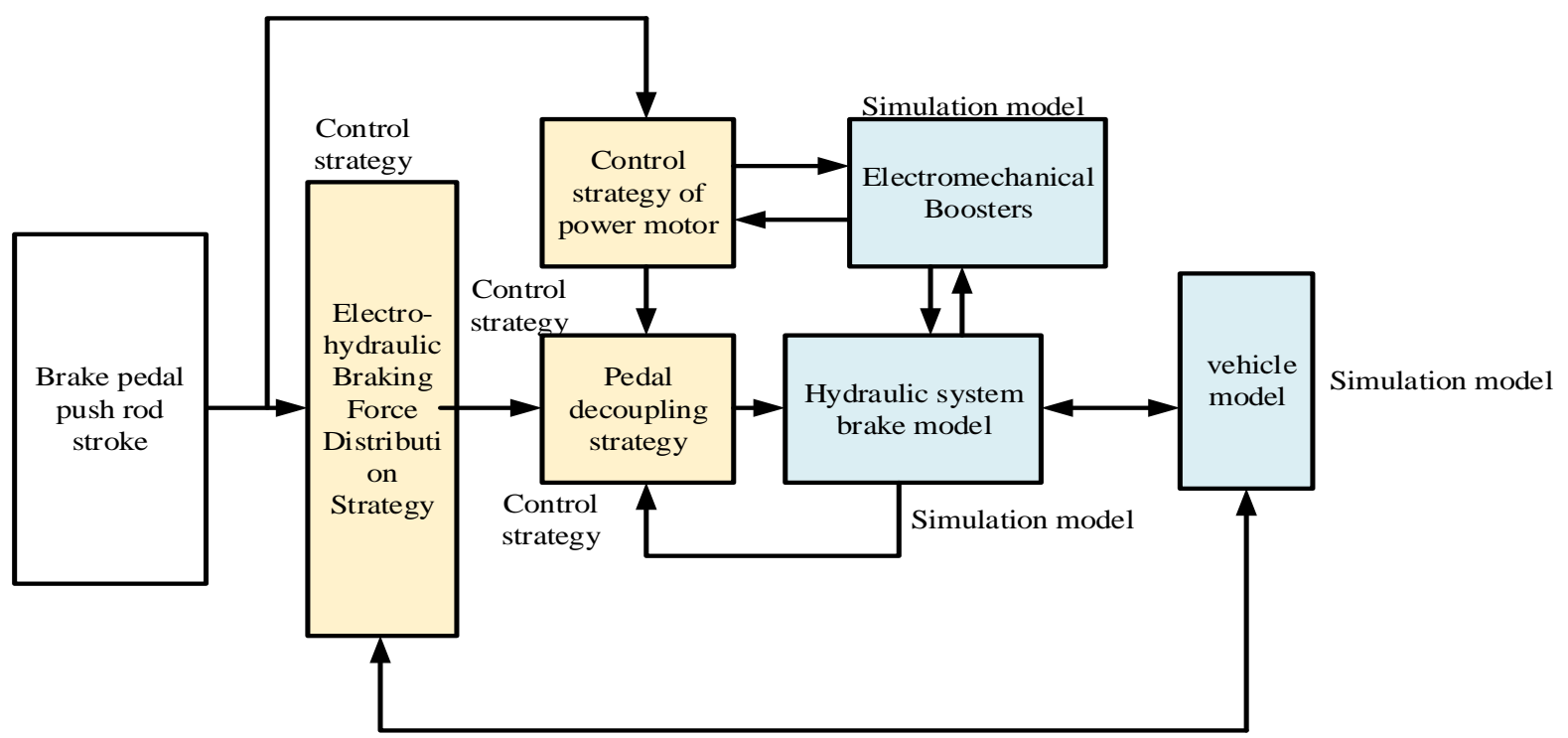

Figure. 4 The overall design block diagrams of the decoupling strategy of the electromechanical booster

\subsection{Co-simulation Model Construction}

To verify the performance and energy consumption characteristics of the composite braking designed, based on the actual parameters of a certain type of new energy vehicle, a composite braking unit model is established using MATLAB/Simulink according to the optimal control strategy of effective feedback power. The curb weight of the selected new energy vehicle is $1200 \mathrm{~m} / \mathrm{kg}$, the windward area is $2.0 \mathrm{~A} / \mathrm{m}^{2}$, the wheel radius is $300 \mathrm{r} / \mathrm{mm}$, and the centre of mass $h_{g}$ is $600 \mathrm{~mm}$. The wheelbase $L$ is $2400 \mathrm{~mm}$, the distance $b$ from the centre of mass to the rear axis is $950 \mathrm{~mm}$, and the transmission ratio $i_{g}$ is 8 . The maximum torque of the motor is $100 \mathrm{~N} . \mathrm{m}$, and the maximum speed of the motor is $10000 \mathrm{r} . \mathrm{min}^{-1}$. The rated capacity of the battery pack is rated at 100A.h, and the rated voltage of the battery pack is $190 \mathrm{~V}$.

The hydraulic brake subsystem model is composed of an integrated brake master cylinder assembly model, a hydraulic adjustment unit model, and a brake wheel cylinder model. To facilitate modelling, the hydraulic brake subsystem model is first divided into actuators and controllers, and then an integrated brake master cylinder assembly model is established. In this work, the wheel cylinder of the disc brake is simplified as a spring damping model. The diameter $d$ of the wheel cylinder of the disc brake is $50 \mathrm{~mm}$, the brake factor is 0.7 , and the brake acting radius $\mathrm{r} / \mathrm{mm}$ is 100 [28]. In the regenerative braking subsystem model, the motor, motor controller, and battery pack all have thermal management systems, and the motor's feedback time is relatively short.

Therefore, the battery pack involved uses the voltage source equivalent internal resistance model to simulate the charge/discharge process of the battery pack [29]. The electric motor is modelled by the average value model, and the charging efficiency of the battery pack and the motor feedback efficiency are obtained from the battery pack charging experiment and the motor bench experiment. The vehicle and test environment sub-models include vehicle model, driver model, and cycle mode model. The input of the model is the running speed of the vehicle and the target speed.

The output is the drive and brake command with a value range of $[0,1]$. The model is then used to verify the pedal decoupling under three braking intensities. The maximum value of the vehicle speed influence factor is set to $15 \mathrm{~km} / \mathrm{h}$, the minimum value to $5 \mathrm{~km} / \mathrm{h}$, the maximum value of the braking intensity influence factor to 0.75 , and the minimum value to 0.55 . The maximum value of the battery impact factor is set to 0.95 , and the minimum value is set to 0.85 .

\section{Verification of Decoupling Strategy based on Electronic Mechanical Booster}

\subsection{Pedal Decoupling under High Braking Intensity}

Figure 5 shows the simulation results of pedal decoupling under high braking intensity. The simulation condition is stepping on the pedal and keeping its braking intensity stable at 0.65 , and the initial speed value of the passenger car is $60 \mathrm{~km} / \mathrm{h}$. Figure 5(a) shows a schematic diagram of the pedal decoupling phase with changes in the degree of braking. Figure 5(b) shows the distribution of electro-hydraulic braking force. In Figure 5(b), Fbref represents the target value of the total braking force of the passenger car, $F_{\text {bref }}$ refers to the target value of the total braking force of the passenger car, $F_{h b r e f}$ 
refers to the target value of the hydraulic braking force, and $F_{r b r e f}$ is the target value of regenerative braking force. In Figure 5(c), $P_{\text {ref }}$ is the target hydraulic pressure of the master cylinder under nonregenerative braking, $P_{\text {mcref }}$ is the target cylinder pressure of the master cylinder under consideration

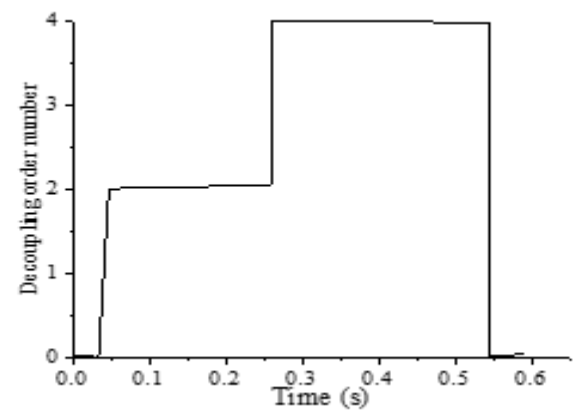

(a)

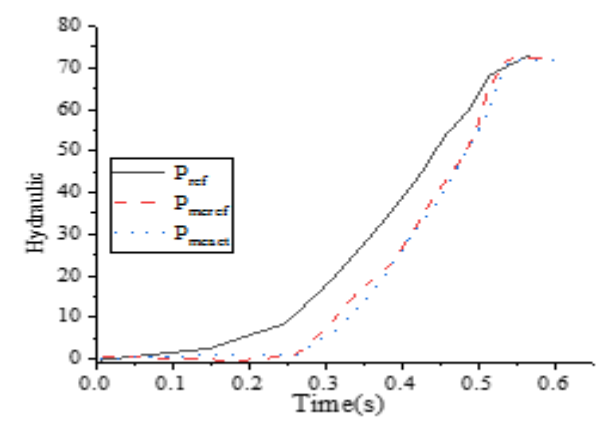

(c) of regenerative braking, and $P_{\text {mcact }}$ is the actual cylinder pressure of the master cylinder under consideration of regenerative braking.

Figure 5(d) shows the magnitude of the hydraulic error of the master cylinder.

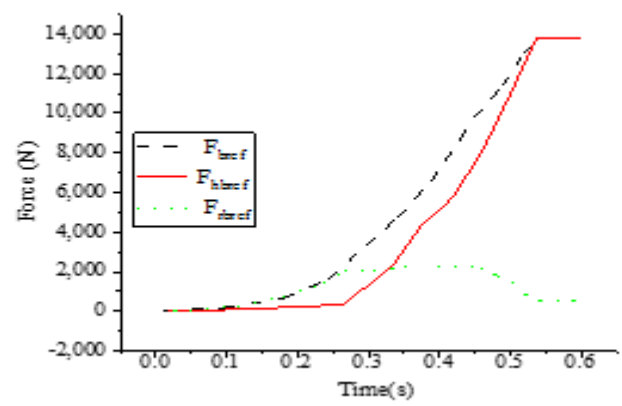

(b)

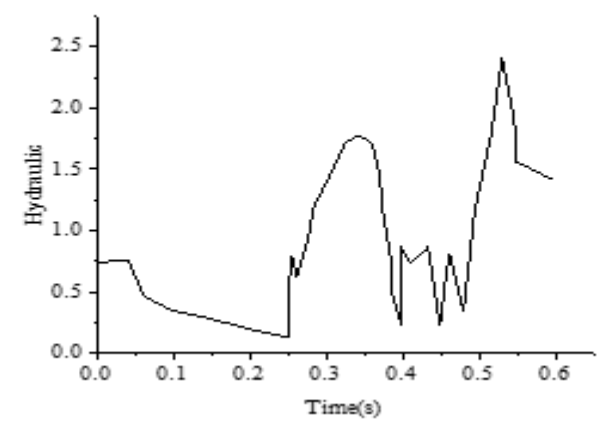

(d)

Figure. 5 Simulation condition of high braking intensity.

(a: decoupling stage, b: electro-hydraulic braking force distribution, c: master cylinder hydraulic pressure, d: master cylinder hydraulic pressure error.)

Figure 5(b) shows that when the braking strength is greater than 0.5 , the regenerative braking force decreases continuously and gradually approaches 0. In Figure 5(c), with the decrease of regenerative braking force, the target hydraulic pressure of the master cylinder in the case of regenerative braking and the total target hydraulic pressure of the master cylinder in the case of no regenerative braking gradually coincide. However, due to the limitation of the motor speed, the actual hydraulic pressure of the master cylinder considering the regenerative braking situation can't immediately keep up with the target hydraulic pressure of the master cylinder, but it can keep up with the target hydraulic pressure change of the master cylinder in the steady state. Figure 5(d) shows that the target hydraulic pressure of the master cylinder is 0 before 0.3 seconds.

According to the decoupling control strategy, in the second stage, the pressure reducing valve opens and the pump doesn't work, and the low-pressure accumulator is regarded as a low-pressure chamber. Therefore, a small pressure drops in the loop.
After the regenerative braking force is gradually withdrawn, the actual hydraulic pressure of the master cylinder increases, but there is a delay and an error within 2 bar of the absolute value compared with the target hydraulic pressure of the master cylinder. The simulation results show that the system designed can adjust the hydraulic pressure of the master cylinder in the decoupling process and meet the demand of decoupling under the larger braking strength.

\subsection{Pedal Decoupling under Medium Braking Intensity}

Figure 6 shows the simulation results of pedal decoupling under medium braking intensity. The simulation condition of medium braking intensity is that the brake pedal performs a forward and return movement. In the experiment, the stable braking intensity is set to 0.4 , and the initial vehicle speed is set to $60 \mathrm{~km} / \mathrm{h}$. 


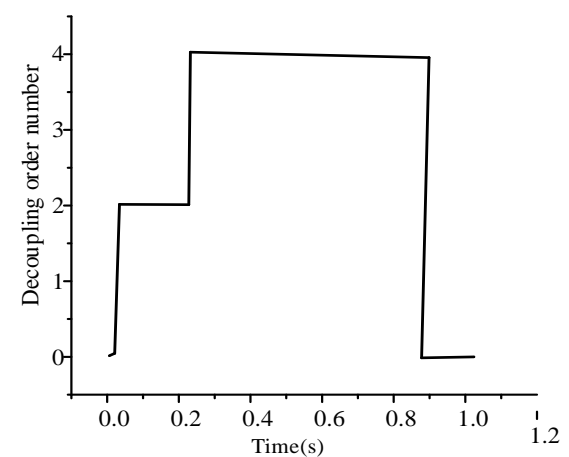

(a)

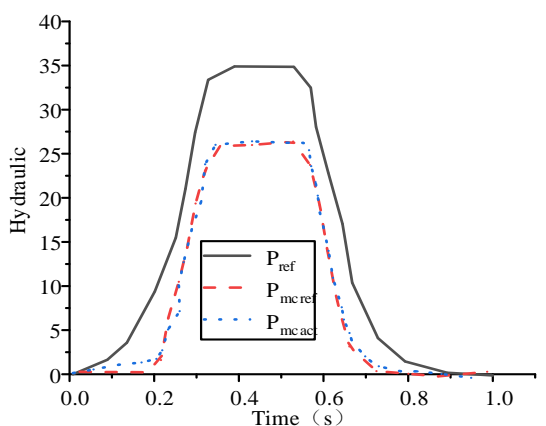

(c)

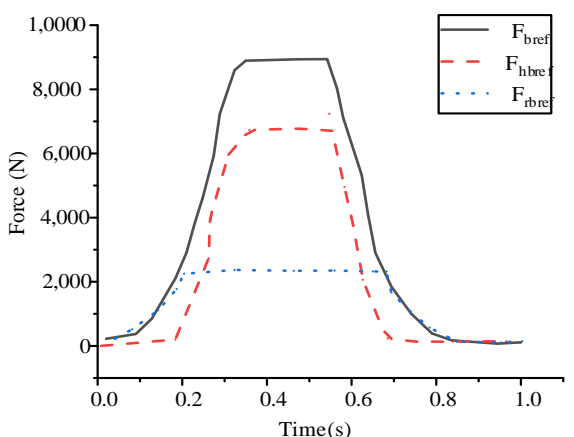

(b)

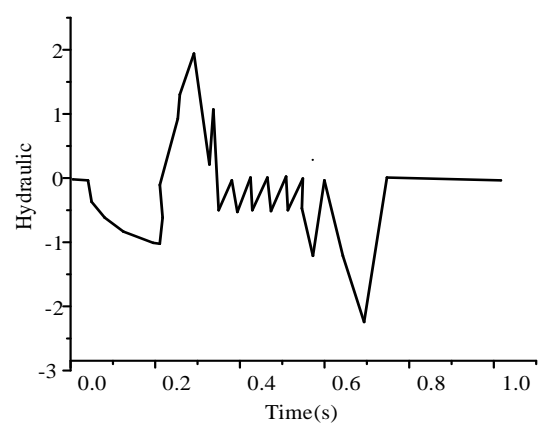

(d)

Figure. 6 Simulation operating conditions under medium braking intensity.

(a: decoupling stage, b: electro-hydraulic braking force distribution, $c$ : master cylinder hydraulic pressure, $d$ : error of master cylinder hydraulic pressure.)

Figure 6(a) shows the decoupling stage when the pedal is decoupled under this working condition. Figure 6(b) shows the electro-hydraulic-braking force distribution. In the second stage, as the stroke of the brake pedal increases, the regenerative braking force also increases. When the regenerative braking force reaches a fixed peak, it enters the third stage. Figure 6(c) shows the change of the master cylinder hydraulic pressure under this working condition. Figure 6(d) shows the hydraulic error of the master cylinder.

Figure 6(c) shows that under this working condition, the regenerative braking force always exists, so the target hydraulic pressure of the master cylinder is always smaller than the total target hydraulic pressure, and the regenerative braking force supplements the lack of hydraulic braking force. In the second stage, the actual hydraulic pressure has a slight increase within the error range, and then enters the third stage. In the third stage, the actual hydraulic pressure is closer to the target hydraulic pressure of the master cylinder.
Figure 6(c) shows that the maximum absolute error of the master cylinder hydraulic pressure under this working condition is 2.5 bar, and it has a small steady-state error.

The experimental results show that the designed system can adjust the hydraulic pressure of the master cylinder during the decoupling process and meet the decoupling requirements under the medium braking intensity.

\subsection{Pedal Decoupling under Low Braking Intensity}

The simulation condition of low braking intensity is performing a forward and return movement of the brake pedal, and the steady braking intensity is set to 0.1 .

Figure 7 is its simulation diagram, Figure $7(\mathrm{c})$ is the master cylinder hydraulic pressure, and Figure $7(d)$ is the master cylinder error. 


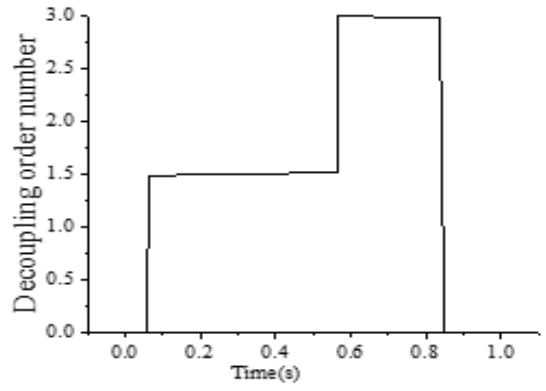

(a)

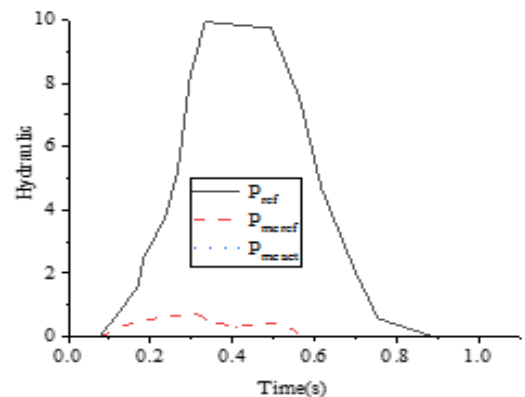

(c)

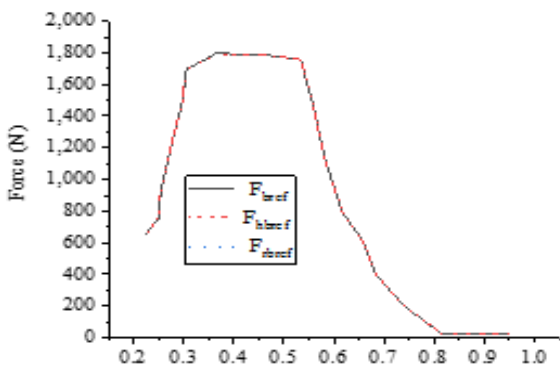

(b)

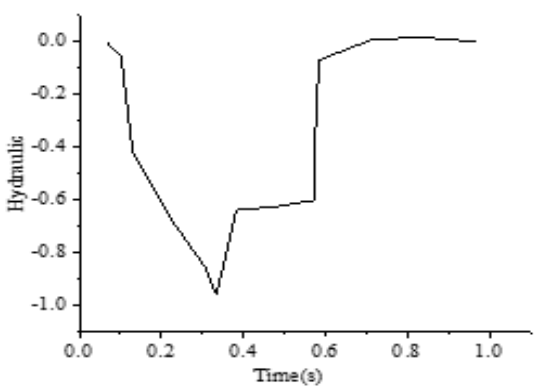

(d)

Figure. 7 Simulation operating conditions under low braking intensity.

(a: decoupling stage, b: electro-hydraulic braking force distribution, c: master cylinder hydraulic pressure, $d$ : error of master cylinder hydraulic pressure.)

Figure 7(a) shows that in the forward stroke and pedal hold phase, only the regenerative braking works. Therefore, the hydraulic pressure needs to be adjusted during the return stroke in the second stage, so it enters the third stage. Figure 7(b) shows that the target total braking force is provided by the target regenerative braking force, and the target hydraulic braking force is zero. Figure 7(c) shows that the absolute value of the master cylinder error is small, and this strategy can meet the decoupling requirements under low braking intensity.

\subsection{Comparative Test of Different Electro- Hydraulic Compound Braking Schemes}

1, 2, and 3 in Figure 8 represent the performance comparison diagrams of decoupled systems using optimal braking force distribution strategy, maximum regenerative braking force distribution strategy, and effective feedback power optimal strategy, respectively. Figure 8 (a)-8(c) show the change in the battery pack, the change in energy efficiency, and the return energy efficiency in turn.

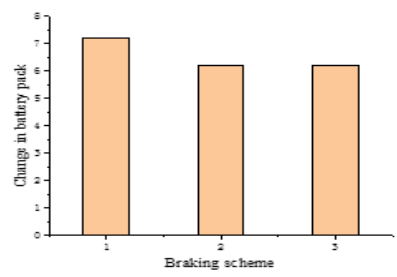

(a)

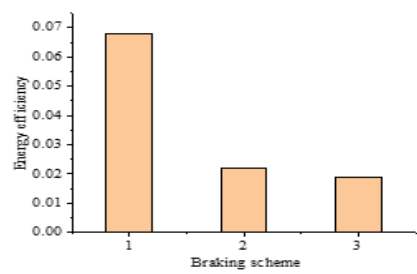

(b)

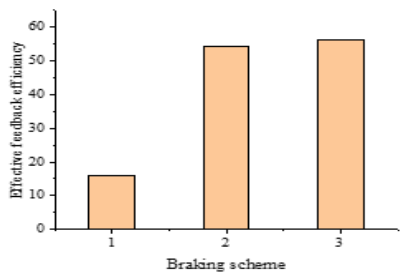

(c)

Figure. 8 Comparison of different electro-hydraulic compound braking schemes 
Figure 8 shows that the use of different control strategies has a great impact on the energy feedback, and the effective feedback efficiency reaches $56 \%$, which is slightly higher than the maximum regenerative braking force scheme.

\section{Discussion}

In this work, the scheme design and control strategy of the new pedal decoupling scheme are developed. In the scheme design, the advantages and disadvantages of different schemes are analysed. In addition, the working principle of the electronic mechanical booster is explained, and the characteristics of the main components are tested. In the control strategy design, the decoupling strategy of the decoupling scheme is studied. When the whole model is established, a reasonable simplification is made according to the requirements of this work. When a decoupling control strategy is designed, the braking force distribution constraints are first studied. Then, the control strategy is designed, and the control strategy of the electronic mechanical booster is studied. Finally, the coordinated decoupling strategy based on electronic mechanical booster is verified through offline simulation.

The experimental results of this research are compared with the those of Wang et al. (2018) [30]. In the simulation experiment under high braking intensity, when the regenerative braking force is gradually withdrawn, the difference between the actual hydraulic pressure of the master cylinder under the constructed decoupling strategy and the target hydraulic pressure of the master cylinder considering the regenerative braking is small, which is about 2 bar. The difference between the actual hydraulic pressure of the master cylinder based on the decoupling strategy proposed by Wang et al. and the target hydraulic pressure of the master cylinder considering regenerative braking is large, about 2.5 bar.

In the simulation experiment under medium braking intensity, when the regenerative braking force is gradually withdrawn, the difference between the actual hydraulic pressure of the master cylinder based on the constructed decoupling strategy and the target hydraulic pressure of the master cylinder considering the regenerative braking is small, which is about 2.5bar. The difference between the actual hydraulic pressure of the master cylinder based on the decoupling strategy proposed by Wang et al. and the target hydraulic pressure of the master cylinder considering the regenerative braking is relatively large, which is about 3 bar. The results prove that the decoupling strategy proposed is of relatively higher adjustment sensitivity and better stability.

\section{Conclusion}

This work conducts research on the pedal decoupling strategy of electronic mechanical assisted braking. First, the pedal decoupling scheme is designed, and the electronic mechanical assisted braking model and the vehicle model are established. Then, the pedal decoupling strategy based on the new decoupling scheme is studied. Finally, simulation and experimental verification are carried out.

The test results show that under the three test conditions, the hydraulic pressure of the master cylinder during the decoupling process can be adjusted to meet the decoupling requirements. The feasibility of the proposed scheme and the effectiveness of the control strategy are verified. However, the scheme designed still has certain shortcomings. In the study of pedal decoupling, only the characteristics of hydraulic pressure changes under different schemes are studied. However, the relationship between the forces at the pedal end during the decoupling process is not been studied in detail, which will be completed in future work.

\section{References}

[1] Padmagirisan P., Sowmya R., Sankaranarayanan V. (2019) Power-assist control of a humanelectric hybrid bicycle with energy regeneration and cruise control. Proceedings of the Institution of Mechanical Engineers, 233(2), 179-191.

[2] Gao X. Y., Wang T., Wang H. F, et al. (2020) Cooling a Mechanical Oscillator in Opto-electromechanical System with Frequency Modulations International Journal of Theoretical Physics, 59(9), 1-14.

[3] Peng J., Togawa T., Tachibana T, et al. (2019) Numerical and Experimental Investigation on Braking Characteristics of an Electro-Rheological (ER) Micro Brake. JFPS International Journal of Fluid Power System, 11(3), 124-129.

[4] Kwon S., Lee S., Lee J, et al. (2019) Accurate State Estimation for Electro-Mechanical Brake Systems Journal of Electrical Engineering and Technology 14(2), 889-896.

[5] Sheng H., Gai J T., Ma T, et al. (2018) Analysis of Coordinated Control of Electro-Mechanical Braking for Tracked Vehicles. Beijing Ligong Daxue Xuebao/Transaction of Beijing Institute of Technology, 38, 195-199.

[6] Bravo R. R. S., Negri V. J. D., Oliveira. A. A. M. (2018) Design and analysis of a parallel hydraulic - pneumatic regenerative braking system for heavy-duty hybrid vehicles. Applied Energy, 225, 60-77. 
[7] Peng X., He L., Lü Y. (2018) Fuzzy sliding mode control based on vehicle slip ratio for electromechanical braking systems. Zhongnan Daxue Xuebao (Ziran Kexue Ban)/Journal of Central South University (Science and Technology), 49(2), 360-370.

[8] Afzal A., Rashid I. A., Shakir H. M. F, et al. (2021) Characterization of the Aniline.DBSA/Thermoplastic Polyurethane Blends for the Thermo-Mechanical and ElectroMechanical Properties. Key Engineering Materials, 875, 96-103.

[9] Phua W. K., Rabeek S. M., Han B, et al. (2020) AINBased MEMS (Micro-Electro-Mechanical System) Hydrophone Sensors for IoT Water Leakage Detection System. Water, 12(11), 2966.

[10] Yu J., Zhang S., Yang W, et al. (2020) Design and Application of Buoy Single Point Mooring System with Electro-Optical-Mechanical (EOM) Cable. Journal of Marine Science and Engineering, 8(9), 672.

[11] Che-Pin C., Mao-Hsiung C. (2018) Mathematical Simulations and Analyses of Proportional ElectroHydraulic Brakes and Anti-Lock Braking Systems in Motorcycles. Actuators, 7(3), 34.

[12] Chen Z., Xia Y., He J, et al. (2020) Elastic-electromechanical modelling and analysis of piezoelectric metamaterial plate with a selfpowered synchronized charge extraction circuit for vibration energy harvesting. Mechanical Systems and Signal Processing, 143, 106824.

[13] Kim Y., Park C., Yu D, et al. (2018) Development of Steering Assist System for Emergency Steering Avoidance. Transactions of the Korean Society of Mechanical Engineers A, 42(5), 437-444.

[14] Wang D. Y., Li D. Q., Chen J, et al. (2019) A Position Synchronization Control for Dual Redundant Electro-Mechanical Actuation in Flight Control System. IOP Conference Series: Materials Science and Engineering, 638, 012007.

[15] Liu Y., Xu J., Shuai Z, et al. (2020) Passivitybased decoupling control strategy of single-phase LCL-type VSRs for harmonics suppression in railway power systems. International Journal of Electrical Power \& Energy Systems, 117, 105698.

[16] Song D., Li Y. (2020) Study on Composite Control Strategy of Transient Air-Fuel Ratio for Gasoline Engine Based on Model. IOP Conference Series: Earth and Environmental Science, 513(1), 012027.

[17] Shen X., Nie C., Lv H, et al. (2020) Study on Hybrid Energy Storage Configuration and Control Strategy of Grid-connected Wind Hydrogen System. E3S Web of Conferences, 2020, 155(5):01003.

[18] He Y., Mai X., Cui C, et al. (2019) Dynamic Modeling, Simulation, and Experimental Verification of a Wafer Handling SCARA Robot with Decoupling Servo Control. IEEE Access, 7, 47143-47153.

[19] Wang Q., Ding Z., Cheng M, et al. (2020) A Parameter-Exempted, High-Performance Power Decoupling Control of Single-Phase Electric Springs. IEEE Access, 8, 1.

[20] Li X., Fang Y., Liu L. (2020) Decoupling Predictive Control of Strip Flatness and Thickness of Tandem Cold Rolling Mills Based on Convolutional Neural Network. IEEE Access, 8, 3656-3667.

[21] Jia R., Wu Y. M, et al. (2019) Decoupling and Optimal Control of Multilevel Buck DC-DC Converters with Inverse System Theory. IEEE Transactions on Industrial Electronics, 67(9), 7861-7870.

[22] Sirotkin E., Yamazaki K., Miroshnichenko A. (2020) Gearbox Development for an Emergency Brake System of the Wind Turbine. IOP Conference Series Earth and Environmental Science, 459, 022010.

[23] Zhao Z., Li M., Wang C, et al. (2019) Dynamic Modeling of Brake in Power-Split DHT and Pressure Tracking Control with Sliding Mode Variable Structure Method. International Journal of Automotive Technology, 20(3), 21-530.

[24] Liu Y., Hao J., Kang P, et al. (2020) Research on dynamic characteristics of compensation mechanism for large-power wind turbine disc brake. Multidiscipline Modeling in Materials and Structures, 16(3), 595-605.

[25] Chen Y. C., Tu C. H., Lin C. L. (2019) Integrated electromagnetic braking/driving control of electric vehicles using fuzzy inference. Electric Power Applications, IET, 13(7), 1014-1021.

[26] Han J. O., Shin J. W., Kim J. C, et al. (2019) Design 2-Speed Transmission for Compact Electric Vehicle Using Dual Brake System. Applied Sciences, 9(9), 1793.

[27] Iqbal H., Yi B. J. (2019) Design of a New Bilayer Multipole Electromagnetic Brake System for a Haptic Interface. Applied Sciences, 9(24), 5394.

[28] Wei C., Hofman T., Caarls E. I, et al. (2019) Zone Model Predictive Control for Battery Thermal Management including Battery Aging and Brake Energy Recovery in Electrified Powertrains ScienceDirect. IFAC-PapersOnLine, 52(5), 303308.

[29] Liang J., Walker P. D., Ruan J, et al. (2019) Gearshift and brake distribution control for regenerative braking in electric vehicles with dual clutch transmission. Mechanism and Machine Theory, 133, 1-22.

[30] Wang C., Zhao W., Li W. (2018) Braking sense consistency strategy of electro-hydraulic composite braking system. Mechanical Systems \& Signal Processing, 109(SEP.), 196-219. 